\title{
Measurement of 0.25-3.2 GeV Antiprotons in the Cosmic Radiation
}

\author{
J. W. Mitchell, ${ }^{1}$ L. M. Barbier, ${ }^{1}$ E. R. Christian, ${ }^{1}$ J. F. Krizmanic, ${ }^{1}$ K. Krombel, ${ }^{1}$ J. F. Ormes, ${ }^{1}$ R. E. Streitmatter, ${ }^{1}$ \\ A. W. Labrador, ${ }^{2}$ A. J. Davis, ${ }^{2}$ R. A. Mewaldt, ${ }^{2}$ S. M. Schindler, ${ }^{2}$ R. L. Golden, ${ }^{3}$ S. J. Stochaj, ${ }^{3}$ W. R. Webber, ${ }^{3}$ \\ W. Menn, ${ }^{4}$ M. Hof, ${ }^{4}$ O. Reimer, ${ }^{4}$ M. Simon, ${ }^{4}$ and I. L. Rasmussen ${ }^{5}$ \\ ${ }^{1}$ NASA Goddard Space Flight Center, Greenbelt, Maryland 20771 \\ ${ }^{2}$ California Institute of Technology, Pasadena, California 91125 \\ ${ }^{3}$ New Mexico State University, Las Cruces, New Mexico 88003 \\ ${ }^{4}$ University of Siegen, Siegen, 57068, Germany \\ ${ }^{5}$ Danish Space Research Institute, Lyngby, Denmark
}

(Received 30 August 1995; revised manuscript received 4 December 1995)

\begin{abstract}
The balloon-borne Isotope Matter-Antimatter Experiment (IMAX) was flown from Lynn Lake, Manitoba, Canada on 16-17 July 1992. Using velocity and magnetic rigidity to determine mass, we have directly measured the abundances of cosmic ray antiprotons and protons in the energy range from 0.25 to $3.2 \mathrm{GeV}$. Both the absolute flux of antiprotons and the antiproton/proton ratio are consistent with recent theoretical work in which antiprotons are produced as secondary products of cosmic ray interactions with the interstellar medium. This consistency implies a lower limit to the antiproton lifetime of $\sim 10^{7} \mathrm{yr}$.

PACS numbers: 98.70.Sa, 14.20.Dh, 95.85.Ry
\end{abstract}

Measurement of the antiproton abundance in the cosmic radiation bears strongly on questions ranging from the possibility of a baryon symmetric universe to characterizing the origin and transport of the cosmic rays. However, the interpretation of cosmic ray antiproton measurements has been very uncertain ever since their discovery by Golden et al. [1]. While antiprotons in the cosmic radiation are expected as "secondary" products of interactions of the primary cosmic radiation, principally protons, with the ambient interstellar medium (ISM) [2-4], the first positive measurements $[1,5,6]$ reported higher antiproton fluxes than predicted by contemporary models of cosmic ray transport. Of the numerous explanations proposed (reviewed in Stephens and Golden [7]), one class assumed that secondary antiprotons are produced by cosmic ray protons and helium which have passed through more matter than implied by measured secondary/primary ratios of heavier elements (e.g., boron/carbon). Others considered "exotic" sources such as the evaporation of primordial black holes, the decay of dark matter, or acceleration in relativistic plasmas. It was also suggested that the excess could be a manifestation of a baryon symmetric cosmology [8]. The largest discrepancy was at $\sim 200 \mathrm{MeV}$ [6], where antiproton production in $p-p$ interactions is heavily suppressed [7,9]; however, later measurements gave corresponding upper limits which were significantly lower $[10,11]$. The Isotope Matter-Antimatter Experiment (IMAX) [12] and other recent experiments [13] were designed to clarify these issues.

The fluxes of antiprotons and protons from $\sim 0.2$ to $3.2 \mathrm{GeV}$ were measured by IMAX using magnetic rigidity, ionization energy loss, and velocity measurements to determine the charge (from energy loss and $\beta$ ) and mass (from $Z, \beta$, and rigidity) of incident particles. Data were taken for $\sim 16 \mathrm{~h}$ at an average altitude of $36 \mathrm{~km}$
( $\sim 5 \mathrm{~g} / \mathrm{cm}^{2}$ of residual atmosphere) in a balloon flight from Lynn Lake, Manitoba, Canada on 16-17 July 1992. Results from $5.32 \times 10^{4} \mathrm{~s}$ are reported here.

The IMAX magnetic spectrometer used a single-coil superconducting magnet [14] with drift chambers (DC) [15] and multiwire proportional chambers (MWPC) [14] giving 20 position measurements (12 DC, 8 MWPC) in the bending direction and 12 (8 DC, 4 MWPC) in the nonbending direction. The most probable maximumdetectable rigidity (MDR), determined by the path integral of the magnetic field and the trajectory resolution, was $200 \mathrm{GV} / c$ for $Z=1$ particles. All events used in the present analysis had an MDR $\geq 50 \mathrm{GV} / c$ and both charge signs were treated identically.

Velocities were measured by a time-of-flight (TOF) system [16] with a flight path of $2.54 \mathrm{~m}$ (giving $\beta_{\mathrm{TOF}}$ ), and two Cherenkov counters (C2 and $\mathrm{C} 3)$ [17] with $n=1.043$ silica-aerogel radiators (giving $\beta_{\mathrm{Ck}}$ ). A third Cherenkov counter (C1) was not used in the current analysis. For $Z=1, \beta=1$ particles, the TOF resolution $(\sigma)$ was $122 \mathrm{ps}$ and the yields from $\mathrm{C} 2$ and $\mathrm{C} 3$ were 11 and 13 photoelectrons. The sum of the signals expected from $\mathrm{C} 2$ and $\mathrm{C} 3$ for a $Z=1, \beta=1$ particle was normalized to 1 . Energy loss was measured by the TOF and scintillators S1 and S2. Agreement was required among the four resulting charge measurements.

Tracking quality is a critical factor in positively identifying antiprotons. Track fits were required to use at least 11 position measurements in the bending direction and 7 in the nonbending direction, and have a reduced $\chi^{2} \leq 4$. To eliminate events in which a hard scatter occurred, agreement was required among the rigidities measured by the complete tracking system and by the upper and lower halves. Antiproton candidates were examined for evidence of scattering, and none had to be rejected. To 
eliminate events with multiple tracks, at most 2 DC layers in either orientation could have hits $>4 \mathrm{~cm}$ from the fitted track, and the positions at the TOF derived from timing and tracking had to agree to $\leq 5 \mathrm{~cm}$.

For antiproton and proton kinetic energies below $2.6 \mathrm{GeV}$, mass was determined using $\beta_{\mathrm{TOF}}$, and $\mathrm{C} 2+\mathrm{C} 3$ was limited to $<0.16\left(\beta_{\mathrm{Ck}}=0.965\right)$, improving discrimination against leptons and mesons. Mass separation using $\beta_{\text {TOF }}$ is illustrated in Fig. 1, with the $\mathrm{C} 2+\mathrm{C} 3$ limit relaxed to $\leq 0.36$ to show the full range of antiproton and proton energies $\leq 3.2 \mathrm{GeV}$.

From 2.6 to $3.2 \mathrm{GeV}, \beta_{\mathrm{Ck}}$ was used. This range $(0.16 \leq \mathrm{C} 2+\mathrm{C} 3 \leq 0.36)$ was chosen to minimize background resulting from downward fluctuations of the Cherenkov signals of leptons and mesons. To eliminate spurious events, agreement was required between $\mathrm{C} 2$ and $\mathrm{C} 3$ and between $\beta_{\mathrm{Ck}}$ and $\beta_{\mathrm{TOF}}$. Figure 2 shows the antiprotons clearly separated from the leptons and mesons, which appear as a band around an ordinate of 1 .

Measured antiproton and proton energies, adjusted for ionization energy loss to the top of the atmosphere (TOA), are reported in three intervals: $0.25-1,1-2.6$, and 2.6-3.2 GeV. Average payload column densities were $18.8 \mathrm{~g} / \mathrm{cm}^{2}$ in and above the instrument $\left(11.6 \mathrm{~g} / \mathrm{cm}^{2}\right.$ above the spectrometer) and $10.7 \mathrm{~g} / \mathrm{cm}^{2}$ below the instrument. Antiprotons and protons with $\sim 175 \mathrm{MeV}$ (TOA) are above the instrumental and geomagnetic cutoffs. However, antiprotons with $\geq 250 \mathrm{MeV}$ (TOA) always exit the full payload with $\geq 70 \mathrm{MeV}$ residual energy and the annihilation corrections are reduced. Mass histograms for the three energy ranges are shown in Fig. 3. Note that the antiprotons are clearly mass resolved.

The numbers of antiprotons and protons detected are given in Table I. The lowest energy antiproton observed

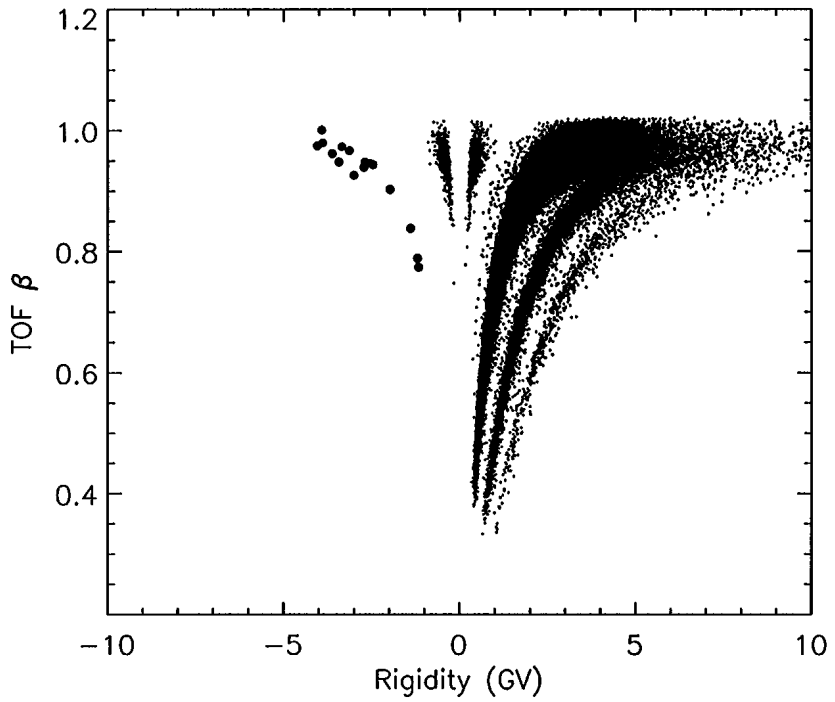

FIG. 1. Velocity determined by the TOF vs rigidity for events with $\mathrm{C} 2+\mathrm{C} 3 \leq 0.36$. The 16 antiprotons have been enhanced (-). Protons, deuterium, and tritium are visible at positive rigidity. The protons and antiprotons are clearly separated from the pions, muons, and electrons. had $583 \mathrm{MeV}$ (TOA). To obtain incident fluxes, the measurements were corrected for backgrounds and losses. Average correction-factors, calculated without spectral weighting, are given below for the three energy intervals ordered from lowest to highest.

Based on a calculation by Stephens [18], we have subtracted $(0.3,1.9,0.7)$ detected antiprotons which are estimated to have come from atmospheric secondary production. In addition, $(0,0,0.5)$ antiprotons have been subtracted based on Monte Carlo simulations of fluctuations in the Cherenkov yields of leptons and mesons. We have also subtracted $\left(1.32 \times 10^{4}, 6.69 \times 10^{3}, 8.44 \times 10^{2}\right)$ detected protons based on a calculation of atmospheric secondaries by Papini, Grimani, and Stephens [19].

Antiprotons or protons undergoing inelastic interactions in or above the instrument are assumed to be lost. Multiplicative corrections for such antiproton losses, calculated using recent antiproton-nucleus annihilation cross sections [20] are $(1.37,1.31,1.29)$ for the instrument and payload and $(1.09,1.08,1.08)$ for the atmosphere. Correction factors for proton losses are $(1.19,1.21,1.21)$ for the payload and $(1.06,1.05,1.06)$ for the atmosphere.

Antiprotons which annihilate below the instrument may also be lost if charged particles produced either directly or from gamma conversion hit the detectors and cause the events to be rejected. Because of the energy dependence of the cross sections and kinematics, this is most probable at low energies. From a GEANT Monte Carlo simulation we estimate corrections of $(1.12,1.06,1.05)$ for this effect, with a maximum of 1.15 at $250 \mathrm{MeV}$. Even if all antiprotons which annihilate below the instrument were

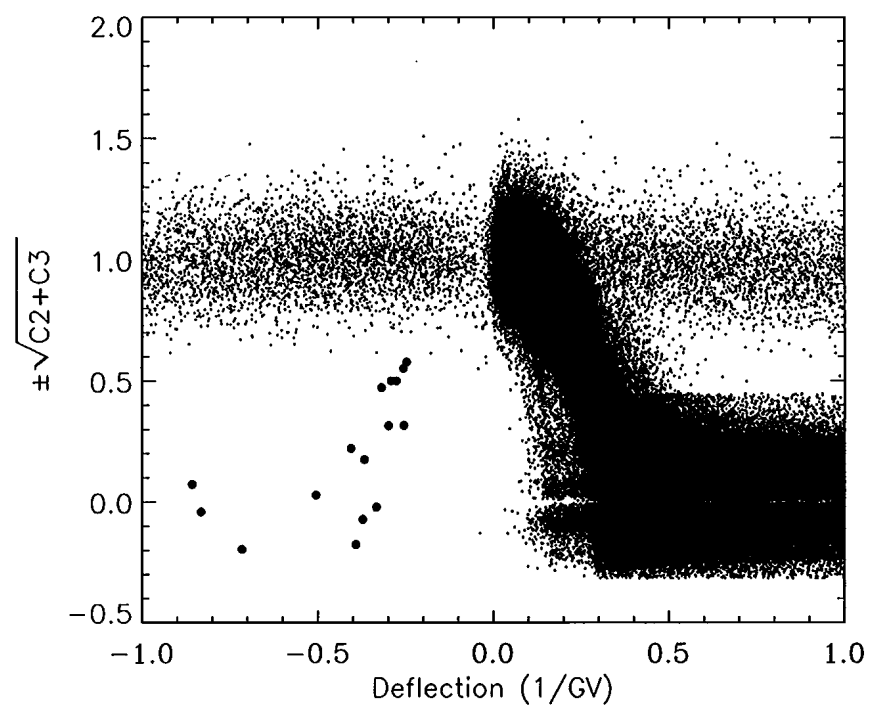

FIG. 2. The signed square root of the absolute value of the $\mathrm{C} 2+\mathrm{C} 3$ signal vs deflection (proportional to rigidity ${ }^{-1}$ ). The 16 antiprotons have been enhanced (O). Low-mass particles occupy the nearly horizontal band. The Cherenkov counter noise of $\sim 0.5$ photoelectron can result in negative values. At low amplitude, fluctuations in the signal are exaggerated by the square root. Below $3.2 \mathrm{GeV}$ (0.6 ordinate) the antiprotons are well separated from background. 
TABLE I. IMAX antiproton and proton fluxes.

\begin{tabular}{cccccc}
\hline \hline $\begin{array}{c}\text { Energy } \\
(\mathrm{GeV})\end{array}$ & $\begin{array}{c}\text { Measured } \\
\text { antiprotons }\end{array}$ & $\begin{array}{c}\text { Measured } \\
\text { protons }\end{array}$ & $\begin{array}{c}\text { TOA antiproton flux } \\
{\left[\left(\mathrm{m}^{2} \mathrm{sr} \mathrm{seV}^{-1}\right]\right.}\end{array}$ & $\begin{array}{c}\text { TOA proton flux } \\
{\left[\left(\mathrm{m}^{2} \mathrm{sr} \mathrm{seV}^{-1}\right]\right.}\end{array}$ & TOA antiproton/proton ratio $^{\text {a }}$ \\
\hline $0.25-1.0$ & 3 & $1.27 \times 10^{5}$ & $2.31_{-1.4}^{+2.5} \times 10^{-2}$ & $7.34 \times 10^{2}$ & $3.14_{-1.9}^{+3.4} \times 10^{-5}$ \\
$1.0-2.6$ & 8 & $1.41 \times 10^{5}$ & $2.11_{-1.0}^{+1.4} \times 10^{-2}$ & $3.94 \times 10^{2}$ & $5.36_{-2.4}^{+3.5} \times 10^{-5}$ \\
$2.6-3.2$ & 5 & $2.31 \times 10^{4}$ & $3.46_{-2.0}^{+3.1} \times 10^{-2}$ & $1.78 \times 10^{2}$ & $1.94_{-1.1}^{+1.8} \times 10^{-4}$ \\
\hline \hline
\end{tabular}

${ }^{\mathrm{a}}$ Quoted error reflects only the statistical uncertainty of the number of measured antiprotons.

lost, these corrections would be at most $(1.17,1.14,1.14)$ and 1.26 at $250 \mathrm{MeV}$.

The geometry factor was $\sim 140 \mathrm{~cm}^{2}$ sr and overall detection efficiency, including live time $(0.74)$, telemetry recovery (0.93), and data selection (0.51), was $\sim 0.35$, giving an effective exposure of $2.6 \times 10^{2} \mathrm{~m}^{2} \mathrm{srs}$. The fluxes of antiprotons and protons corrected to the top of the atmosphere are given in Table I. Systematic uncertainties in the antiproton flux are $\sim 10 \%$, due primarily to uncertainties in the atmospheric background corrections and, at the very lowest energies, in the annihilation corrections. Systematic uncertainties in the proton flux are $\sim 3 \%$.

To derive theoretical predictions of $1 \mathrm{AU}$ fluxes, we have applied a spherically symmetric solar modulation model [21] to the interstellar antiproton spectra of Webber and Potgieter (WP) [3] and Gaisser and Schaefer (GS) [4]. The modulation parameter, $\phi=750 \mathrm{MV}$, was chosen to

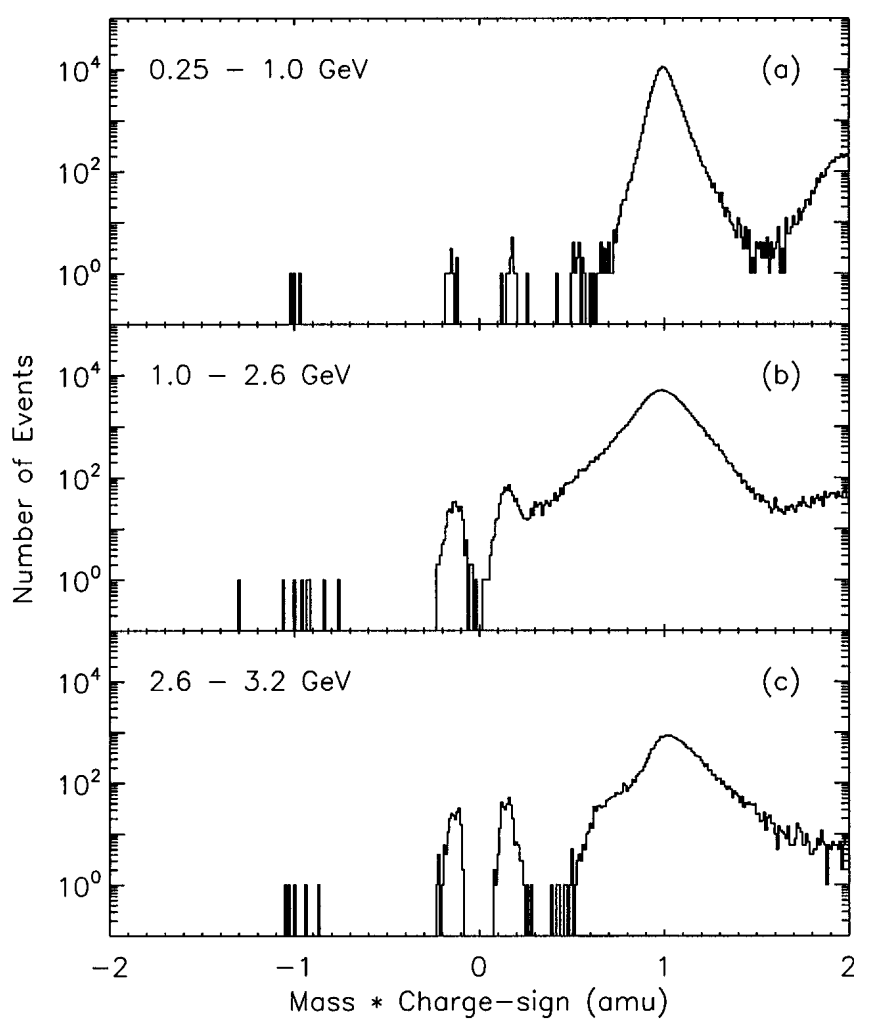

FIG. 3. Mass $(\mathrm{amu}) \times$ charge sign for the three energy intervals. The antiprotons are well resolved in mass and clearly separated from the leptons and mesons. make the modulated WP interstellar proton spectrum and the IMAX proton spectrum agree.

Historically, antiproton measurements have been reported as the ratio of the flux (or flux limit) of antiprotons to that of protons. In Fig. 4, the IMAX measurements of this ratio are compared with previous results, with theoretical limits derived by GS, and with the ratio of the modulated WP spectra. Note that below $\sim 2 \mathrm{GeV}$ the ratio is expected to vary with solar modulation [3,22]. The earlier results of Bogomolov et al. [5] (based on one antiproton detected from 0.2 to $2 \mathrm{GeV}$ and three from 2 to $5 \mathrm{GeV}$ ) and the low-energy upper limits $[10,11]$ are consistent with the IMAX results. The IMAX measurements are also in agreement with the most recent theoretical predictions [3,4], which are higher than in the earlier literature (see [7]). Note that the measurements and predictions now have comparable uncertainties.

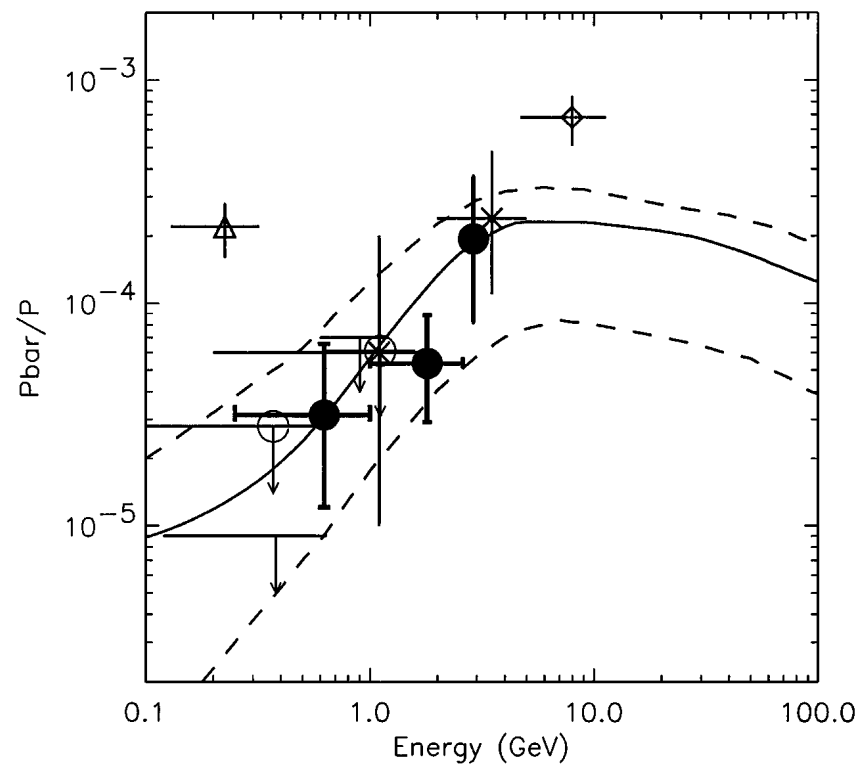

FIG. 4. The TOA antiproton/proton ratios measured by IMAX compared with previous measurements, with limits to the $1 \mathrm{AU}$ ratio calculated by GS [4] for 1989 (dashed lines), and with the ratio obtained by modulating WP [3] interstellar spectra to 1992 conditions (solid line). The points are IMAX (bold, filled circles), Golden et al. [1] (open diamond), Bogomolov et al. [5] (asterisk), Buffington et al. [6] (open triangle), Stochaj [11] (no symbol), and Salamon et al. [10] (open circle). Note that the higher-energy upper limit of [10] and the low-energy point of [5] coincide. 


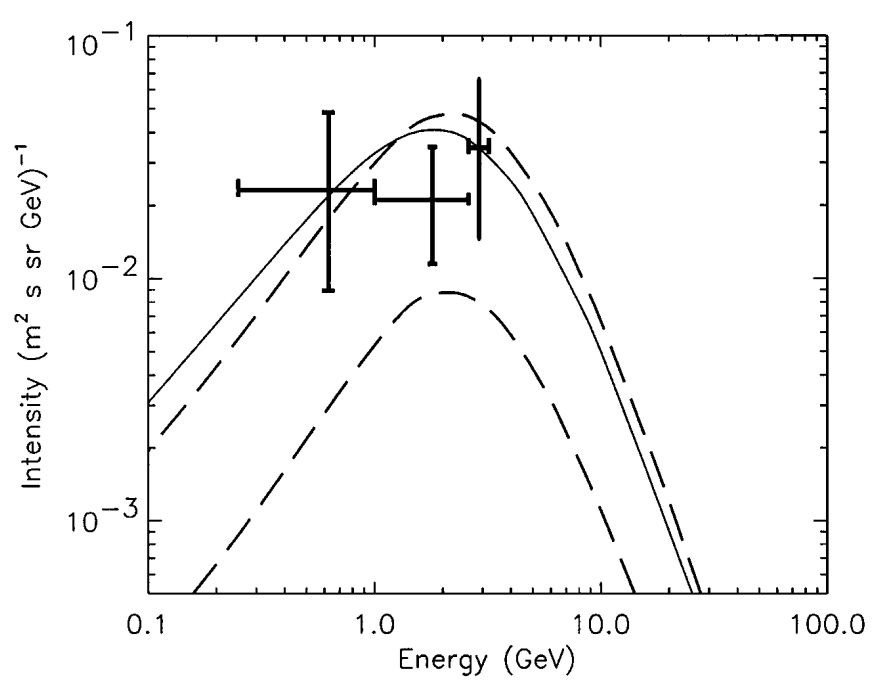

FIG. 5. The TOA flux of antiprotons measured by IMAX together with the WP [3] antiproton spectrum (solid line) and the GS [4] spectral limits (dashed lines), modulated to 1992 levels.

If the lowest energy interval is divided at $0.5 \mathrm{GeV}$, the $0.25-0.5 \mathrm{GeV}$ fluxes [in $\left(\mathrm{m}^{2} \mathrm{srs} \mathrm{GeV}\right)^{-1}$ ] would be $8.3 \times 10^{2}$ for protons and $<6.2 \times 10^{-2}$ for antiprotons $(86 \%$ confidence level), while the $0.5-$ $1 \mathrm{GeV}$ fluxes would be $7.0 \times 10^{2}$ for protons and $3.1_{-1.9}^{+3.4} \times 10^{-2}$ for antiprotons. The corresponding antiproton/proton ratios would be $<7.3 \times 10^{-5}(86 \% \mathrm{CL}$ as in $[10,11])$ for $0.25-0.5 \mathrm{GeV}$ and $4.5_{-2.7}^{+4.8} \times 10^{-5}$ for $0.5-1 \mathrm{GeV}$

In Fig. 5, the IMAX measurements of the antiproton flux at $1 \mathrm{AU}$ are compared to the modulated WP and GS spectra. The measurements are consistent with the WP flux and fall within the maximum and minimum GS fluxes. Averaged over $0.25-3.2 \mathrm{GeV}$, the WP calculations give a TOA antiproton flux [in $\left(\mathrm{m}^{2} \mathrm{srs} \mathrm{GeV}\right)^{-1}$ ] of $3.4 \times 10^{-2}$, while the range of GS spectra predict $1.6_{-0.9}^{+2.2} \times 10^{-2}$. The total observed IMAX antiproton flux of $2.5_{-0.8}^{+1} \times 10^{-2}$ is consistent with both calculations.

We conclude that within the uncertainties of the current calculations, the antiproton fluxes measured by IMAX are consistent with cosmic ray antiprotons in this energy range being dominated by secondaries of the primary cosmic radiation. We find no need for exotic sources of antiprotons to explain our measurements. The present IMAX results are the first to clearly establish that the bulk of cosmic ray antiprotons below $3 \mathrm{GeV}$ have a secondary origin. This implies that the antiproton lifetime must be comparable to or greater than the storage lifetime of cosmic rays in the galaxy [23], estimated from the abundance of ${ }^{10} \mathrm{Be}$ to be $\sim 10^{7} \mathrm{yr}$ [24].

We thank Glen Albritton, Barbara Kimbell Golden, Steve Holder, Bob Hull, Roy Park, and Don Righter for dedicated technical support; Francesco Cafagna (U. Bari, Italy) and Heather Muise for help with GEANT; and the National Scientific Balloon Facility for the IMAX flight. IMAX was supported in the U.S. by NASA RTOP 353-87-02 (GSFC) and Grants NAGW-1919 (Caltech) and NAGW-1418 (NMSU) and in Germany by DARA 50QV9191 and DFG Si-290/7.

[1] R. L. Golden et al., Phys. Rev. Lett. 43, 1196 (1979); R. L. Golden et al., Astrophys. Lett. 24, 75 (1984).

[2] T. K. Gaisser and R. H. Maurer, Phys. Rev. Lett. 30, 1264 (1973).

[3] W. R. Webber and M. S. Potgieter, Astrophy. J. 344, 779 (1989).

[4] T. K. Gaisser and R. K. Schaefer, Astrophy. J. 394, 174 (1992).

[5] E. A. Bogomolov et al., in Proc. 16th Int. Cosmic Ray Conf., Kyoto 1, 330 (1979); E. A. Bogomolov et al., in Proc. 20th Int. Cos. Ray Conf., Moscow 2, 72 (1987); E. A. Bogomolov et al., in Proc. 21st. Int. Cos. Ray Conf., Adelaide 3, 288 (1990).

[6] A. Buffington, S.M. Schindler, and C. Pennypacker, Astrophy. J. 248, 1179 (1981); A. Buffington and S. M. Schindler, Astrophy. J. 248, L105 (1981).

[7] S. A. Stephens and R. L. Golden, Space Science Rev. 46, 31 (1987).

[8] F. W. Stecker, in Progress in Cosmology, edited by A. W. Wolfendale (D. Reidel Publishing, Dordrecht, 1982), p. 1.

[9] M. Simon and U. Heinbach, in Cosmic Rays, Supernovae and the Interstellar Medium, edited by M. M. Shapiro et al. (Kluwer Academic Publishers, Dordrecht, 1991), p. 137.

[10] S. P. Ahlen et al., Phys. Rev. Lett. 61, 145 (1988); M. H. Salamon et al., Astrophy. J. 349, 78 (1990).

[11] S. J. Stochaj, Ph.D. thesis, University of Maryland, 1990; R. E. Streitmatter et al., Adv. Space Research 9, 1265 (1989).

[12] J. W. Mitchell et al., in Proc. 23rd Int. Cosmic Ray Conf., Calgary 1, 519 (1993).

[13] M. Hof et al., in Proc. 24th Int. Cosmic Ray Conf., Rome (to be published); S. Orito et al., ibid.; G. Barbiellini et al., ibid.

[14] R. L. Golden et al., Nucl. Instrum. Methods 148, 179 (1978); R. L. Golden et al., Nucl. Instrum. Methods Phys. Res., Sect. A 306, 366 (1991).

[15] M. Hof et al., Nucl. Instrum. Methods Phys. Res., Sect. A 345, 561 (1994); W. Menn et al., in Proc. 23rd Int. Cosmic Ray Conf., Calgary 2, 548 (1993).

[16] J. W. Mitchell et al., in Proc. 23rd Int. Cosmic Ray Conf., Calgary 2, 627 (1993).

[17] A. W. Labrador et al., in Proc. 23rd Int. Cosmic Ray Conf., Calgary 2, 524 (1993).

[18] S. A. Stephens, in Proc. 23rd Int. Cosmic Ray Conf., Calgary 2, 144 (1993).

[19] P. Papini, C. Grimani, and S. A. Stephens, in Proc. 23rd Int. Cosmic Ray Conf., Calgary 3, 761 (1993).

[20] V.F. Kuzichev, Yu. B. Lepikhin, and V. A. Smirnitsky, Nucl. Phys. A576, 581 (1994).

[21] L. A. Fisk, J. Geophy. Res. 76, 221 (1971).

[22] A. W. Labrador and R. A. Mewaldt, in Proc. 24th Int. Cosmic Ray Conf., Rome (to be published).

[23] G. Steigman, Astrophy. J. 217, L131 (1977).

[24] M. Garcia-Munoz and J. A. Simpson, Space Sci. Rev. 46, 205 (1988). 